\title{
Japanese Activities to bring online academic meetings against COVID-19
}

\author{
How We Learned to Stop Worrying and Love the Online Meetings \\ Hiroki Kashiwazaki \\ reo_kashiwazaki@nii.ac.jp \\ National Institute of Informatics \\ Tokyo, Japan \\ Yusuke Komiya \\ komiyama@nii.ac.jp \\ National Institute of Informatics \\ Tokyo, Japan
Shiu Sakashita
ss@acutus.co.jp
Tokyo, Japan \\ Acutus Software Inc. \\ Takuro Ozaki \\ ozaki@cc.osaka-kyoiku.ac.jp \\ Osaka Kyoiku University \\ Osaka, Japan \\ Eisaku Sakane \\ sakane@nii.ac.jp \\ National Institute of Informatics \\ Tokyo, Japan
Nariyoshi Yamai
nyamai@cc.tuat.ac.jp
Technology
Japan \\ Tokyo University of Agriculture and \\ Hajime Shimada \\ shimada@itc.nagoya-u.ac.jp \\ Nagoya University \\ Aichi, Japan \\ Kazuhiro Mishima \\ three@cc.tuat.ac.jp \\ Tokyo University of Agriculture and \\ Technology \\ Tokyo, Japan \\ Yoshiaki Kitaguchi \\ kitaguchi@gsic.titech.ac.jp \\ Tokyo Institute of Technology \\ Tokyo, Japan
}
Kensuke Miyashita
miyasita@kyoto-wu.ac.jp
Kyoto Women's University
Kyoto, Japan

\begin{abstract}
Various rally events in Japan were canceled or postponed since midFebruary 2020 due to the impact of the new coronavirus COVID-19 which was confirmed in late 2019. In Japan, February and March are the months when many conferences and research meetings are held. The authors were key-persons to bring the conference/research meeting online from late February to mid-March. Over 100 to 1,000 participants attended each conference. Each conference consists of a single session to a maximum of 13 parallel sessions. This paper discusses the prospect of the conference and summarizes the decision-making process and technical decisions of the conferences.
\end{abstract}

\section{KEYWORDS}

video conference, academic meeting, covid-19

ACM Reference Format:

Hiroki Kashiwazaki, Takuro Ozaki, Hajime Shimada, Yusuke Komiya, Eisaku Sakane, Kazuhiro Mishima, Shiu Sakashita, Nariyoshi Yamai, Yoshiaki Kitaguchi, and Kensuke Miyashita. 2021. Japanese Activities to bring online academic meetings against COVID-19: How We Learned to Stop Worrying and Love the Online Meetings. In ACM SIGUCCS Annual Conference

This work is licensed under a Creative Commons Attribution International 4.0 License.

SIGUCCS '20, March 14-April 30, 2021, Virtual Event, USA 2021. ACM ISBN 978-1-4503-8141-3/21/03.

https://doi.org/10.1145/3419944.3441174
(SIGUCCS '21), March 14-April 30, 2021, Virtual Event, USA. ACM, New York, NY, USA, 6 pages. https://doi.org/10.1145/3419944.3441174

\section{TIMELINE OF COVID-19 IN JAPAN}

Novel Coronavirus Infection (COVID-19) is an emerging infectious disease first detected in Wuhan, China, in November $2019^{1}$. As of 23 November 2020, there were 58,229,138 COVID-19 positive cases and 1,382,106 confirmed deaths worldwide, and the number of countries and regions with confirmed infections The first Japanese cases were confirmed on 16 January 2020, followed by a city blockade in Wuhan, China, the following week. In Japan, reports of a COVID outbreak on the cruise ship Diamond Princess ${ }^{2}$ began in late January, and then When the ship entered the port of Yokohama on 3 February, it was placed under quarantine. The risk of infection spread was considered high when all three conditions occurred at the same time: a closed space with poor ventilation, a large number of people in close proximity, and conversations and vocalizations within arms' reach of each other ${ }^{3}$. For this reason, postponement or cancellation of IT events where many participants are concentrated in one place were announced worldwide in starting in early February 2020.

\footnotetext{
${ }^{1}$ Coronavirus disease 2019

https://www.who.int/emergencies/diseases/novel-coronavirus-2019

${ }^{2}$ Diamond Princess - Cruise Ship Information - Princess Cruises

https://www.princess.com/ships-and-experience/ships/di-diamond-princess

${ }^{3}$ Avoid the "Three Cs"!

https://www.mhlw.go.jp/content/10900000/000615287.pdf
} 
Hiroki Kashiwazaki, Takuro Ozaki, Hajime Shimada, Yusuke Komiya, Eisaku Sakane, Kazuhiro Mishima, Shiu Sakashita, Nariyoshi Yamai, Yoshiaki Kitaguchi, and Kensuke SIGUCCS '20, March 14-April 30, 2021, Virtual Event, USA

Mobile World Congress (MWC) ${ }^{4}$, one of the world's largest mobile phone congresses, is usually held in late February in Barcelona, Spain, but on February 5, LG Electronics ${ }^{5}$, one of the major exhibitors, announced the cancellation of its participation in the congress $^{6}$, and on February 7, NVIDIA ${ }^{7}$ announced that it would not be participating in the congress ${ }^{8}$. The GSMA announced that it would not send a delegation to MWC2020. Under these circumstances, the GSMA, the organizer of MWC2020, decided to cancel the MWC2020 on the 12th. The numbers of announcements of cancellations and postponements of IT events increased in Japan as well. On February 16 , in response to this activity, Twitter user potato $4 \mathrm{~d}$ published a Google Spreadsheet ${ }^{9}$, which anyone can edit, to consolidate the status of IT-related events' cancellations and postponements ${ }^{10}$.

On February 20, Japanese Government announced that "Event organizers are requested to reconsider the necessity of holding the event from the viewpoint of preventing the spread of infection, taking into consideration the spread of infection and the situation at the venue. At that time, the Japanese government is not requesting a uniformed refrain from holding events". This statement had a great influence on the direction of the holding of academic events in Japan.

Various academic events are held in March because the graduation and graduate school entrance examinations occur in January and February in Japan, and the entrance examinations happen in February and March. The authors of this paper decided to hold the academic event online, which was scheduled to be held in early March. This paper describes the decision, the preparations for its operation and the production run. It discusses the preparations that need or the preconceived notions that need to be revisited for the large scale of fragmentation that may occur in the future.

\section{COMPARISON OF ONLINE CONFERENCING SYSTEMS}

Some of the most popular online conferencing tools are as follows.

- Cisco Webex Meetings ${ }^{11}$

- Zoom Meetings ${ }^{12}$

- Microsoft Teams ${ }^{13}$

- Google Meet ${ }^{14}$

Most of these online conferencing tools establish online meetings through a server that coordinates communications from the terminal where the online meeting originated. Currently, the main

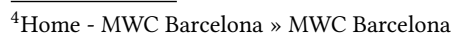

https://www.mwcbarcelona.com

${ }^{5}$ https://www.lg.com

6STATEMENT REGARDING LG ELECTRONICS' PARTICIPATION IN MOBILE WORLD CONGRESS (MWC) $2020 \mid$ LG Newsroom

http://www.lgnewsroom.com/2020/02/statement-regarding-lg-electronics-

participation-in-mobile-world-congress-mwc-2020

${ }^{7}$ https://www.nvidia.com

${ }^{8}$ Update on MWC Barcelona | NVIDIA Blog

https://blogs.nvidia.com/blog/2020/02/07/mwc-barcelona-update

${ }^{9}$ National Tech Conferences on Coronaviruses 2020 (Japanese)

https://docs.google.com/spreadsheets/d/1nGtJBgrnAZBEWL26ATguEacAc0q7btkMAQ

CoiZlYUw4

${ }^{10} \mathrm{https} / / /$ twitter.com/potato4d/status/1228934673604067328 (Japanese)

${ }^{11}$ https://www.webex.com/

${ }^{12}$ https://zoom.us/meetings

${ }^{13} \mathrm{https} / /$ www.microsoft.com/en-us/microsoft-365/microsoft-teams/group-chatsoftware

${ }^{14}$ https://meet.google.com/
}

configuration method of these servers is to provide a cloud server. The features descriptions are below.

Cisco Webex Meetings was an online conferencing tool developed by WebEx. It was acquired by Cisco Systems in 2007 and consolidated with Cisco's Cisco Spark offering, which also provided the conferencing hardware. A unique feature is the ability to convert the recorded content into text (transcription). Zoom Meetings is an online conferencing tool launched in 2003, with subsequent enhancements that allow integration with other tools such as Polycom, Slack, and Google Calendar. The number of users of Zoom is rapidly increasing. The issues of vulnerability discovery, security, and privacy was discussed.

Microsoft Teams is a communication tool in Microsoft's collaborative application suite, Office 365 , rather than a standalone online conferencing tool. Google Meet is a service provided by Google, initially called Hangouts, and was free to use. Later, it reorganized into Hangouts Chat, a messaging service, and Hangouts Meet, an online conferencing tool, which became part of the G Suite of cloud services for businesses.

Although there is a wide range of prices available for each system, to run an online academic research conference, we mainly list the systems that can accommodate more than 100 participants.

- Webex Meetings: $\$ 20$ per month for a Plus host account for up to 100 people, and $\$ 30$ per month for a Business account for up to 200 people

- Zoom: $\$ 20$ per month for a Pro host account for up to 100 people, and $\$ 25$ per month for a Business host account for up to 300 people

- Teams: $\$ 5$ per user per month. However, it is provided as a tool in Office 365 Business Essentials, and is available for an annual subscription only.

- Google Meet: \$6 per user per month. It is provided as a tool included in G Suite Basic, with a maximum of 100 participants. $\$ 13$ per month for Business with up to 150 participants, and $\$ 30$ per month for Enterprise with up to 250 participants.

\section{CHARACTERISTICS OF INDIVIDUAL INITIATIVES}

This chapter describes four academic events held from late February to mid-March that went online. First, the national conference of the Information and Communication Society will be discussed, followed by the IA/IOT/SITE Joint Workshop, DEIM 2020, and the 82nd National Conference of the Information Processing Society.

\subsection{National Convention of CIS}

In February 2020, the Japan Association for Communication, Information and Society ${ }^{15}$ held its 17 th National Convention at Nagasaki University from February 29, 2020 to March 1, 2020 ${ }^{16}$. In the deliberation, it was resolved that "we will consider the cancellation of the national meeting", "if you are not feeling well, you should not participate in the meeting", and "we will take measures to prevent infection at the venue".

\footnotetext{
${ }^{15}$ Japan Association for Communication, Information and Society (Japanese) http://www.cis.gr.jp

${ }^{16} 17$ th Annual Conference of CIS (Japanese)

http://www.cis.gr.jp/zenkoku.html\#zenkoku17
} 
One of the author, Takuro Ozaki joined the local organizing committee of the convention, and the frequency of discussions increased rapidly since then. For a while, we communicated with each other by e-mail, but the frequency of small adjustments was too much, so we decided to use Cisco Webex Teams for small exchanges. Since we can exchange conversations like a chat room, it is not difficult to get used to the operation, and our discussions have accelerated since we moved to Webex Teams as a discussion tool.

When using Web conferencing systems, it is important to consider the connection environment of the presenter, chairperson, and participants (audience) as well as the sender. For users who have never used a web conferencing system before, there is an urgent need to present a document that enables them to connect without difficulty. Since February 21, when we received the request to operate the online research group, we have been compiling operational tips from the viewpoint of both online management and online presentation, which were published on February 22. The document is organized as follows.

- Web conferencing system overview

- About the online session (overview)

- Overall Preparations

- Environment to be prepared (operator)

- Environment to be prepared (presenter, time of presentation)

- How to participate in the online session (presenter and audience)

- How to present in an online session (presenter)

- How to listen to the online session (audience)

The first keynote address was given on February 29, the first day of the National Convention. In the keynote speech, we received the video clip information for webcasting from the speaker beforehand, so we distributed the data as it was. We were able to deliver the

There were about 10 to 20 participants per venue. Figure 1 shows the presentation at the virtual venue where the author participated as a timekeeper. In particular, we did not find any cases where the audio or video was interrupted, or the session collapsed in the middle.

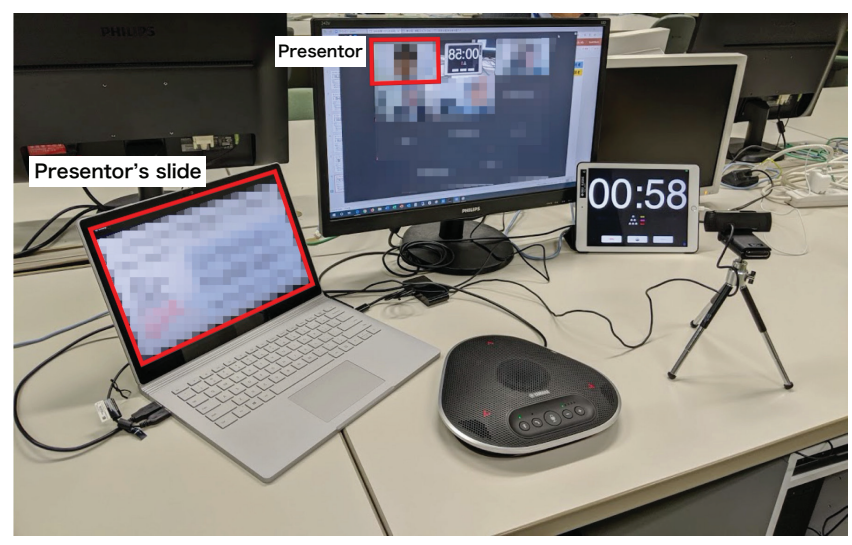

Figure 1: Viewing in the virtual room (and timekeeper)

Although several people from the same room (with seating for about 100 people) participated in the same session at Nagasaki University, each participant used his or her own earphones and microphones, and tried to talk directly with other participants in the room. This effort resulted in the participants sharing the same sound and image in the same virtual venue.

Although the meeting was held in a hurried manner, a questionnaire was sent to the participants, and the answers to the questionnaire were announced at the end of the second day of the national meeting. We received responses from 17 out of 44 people who were guided to the virtual venue.

In addition, a large number of presentations were made from laptops, and a large number of presentations were made remotely from home at the end of February 2020 due to the fact that workshops and other events were beginning to be cancelled and the responses to business trips were varied among universities. Most of the participants from the remote areas came from single or small group rooms, while the participants from the local area participated from a large room such as a conference room. From the free comments in the questionnaire, we received comments such as

- I was confused by the question-and-answer procedure.

- I didn't understand the question-and-answer procedure.

- I was worried about the small number of questions.

Also, we received comments such as "It was difficult to receive the praise at the end of the session in the virtual room, which would have been more natural in the real room". However, just before the National Convention, there was a movement to include a question and answer session, and it was decided to hold a question and answer session as an improvisation.

Since we were able to prepare participants for the preparation of the manual, it is important to prepare participants for events in which they have no experience, for example, in the form of a pre-conference in the form of a "practice session," or in the form of We believe that the preparation of the training video to grasp the image of participation will contribute to lowering the threshold for first-time participants and widen the scope of participants when a similar event occurs in the future.

\section{$3.2 \quad$ IA/IOT/SITE Joint Research Meeting}

The Information Processing Society of Japan (IPSJ) Special Interest Group of the Internet and Operation Technology (IOT), the Institute of Electronics, Information and Communication Engineers (IEICE) Technical Committee on Internet Architecture (IA), and Technical Committee on Social Implications of Technology and Information Ethics (SITE) have held a joint meeting every March, and the 2020 meeting was scheduled to be held on March 2-3 at Nagoya University.

Two weeks before the meeting, we chose Zoom as an online meeting tool because SIG-IOT HQ have already used it for a long time. We firstly consider requirements for online academic meeting and

(1) Support for stand-alone presenter who cannot have enough support at local (-> prepare enough training schedule),

(2) How to avoid copyright violation such as allowed on onsite talk but not allow on broadcasting (-> announce warning about that point),

(3) Keep discussion for a while after online (-> combining Slack utilization). 
Hiroki Kashiwazaki, Takuro Ozaki, Hajime Shimada, Yusuke Komiya, Eisaku Sakane, Kazuhiro Mishima, Shiu Sakashita, Nariyoshi Yamai, Yoshiaki Kitaguchi, and Kensuke SIGUCCS '20, March 14-April 30, 2021, Virtual Event, USA

At this point, we have considered to open as a hybrid (onsite and online) meeting. March is the end of the financial year in Japan so we have not considered extending schedule because some presenters change affiliation at the end of the financial year. We have further discussed including above 1. to 3. points. Final decisions are written after right-allow notation respectively.

(4) How to treat awarding largely depending on evaluation on presentation (-> treating only real-time presenter as awarding candidates).

(5) How to treat onsite registration because meeting registration system consider to terminate pre-registration some days before meeting day (-> did not allow onsite registration)

We also defined that we have to announce the decision at least 1 weeks before considering rules for stockholders' meeting.

1 week before the meeting, we decided to turn to a full online meeting due to the dramatic increase of COVID-19 patients. Major problems are already discussed at hybrid meetings so that we only have to consider where to create HQ and modify the program to a single session to ease operation.

Here's knowledge obtained from this operation. Firstly, we introduce features to improve operability.

- We prepared a timer at a web-cam window on Zoom with a HDMI capture device to ease recognition time elapses for the presenter.

- We prepared a guidebook to avoid race conditions of input devices between Zoom and PowerPoint.

- Chat log of Zoom is unavailable after the meeting so we announced "use Slack for discussion" frequently. Secondly, we introduce confronted troubles including troubles at other meetings.

- Howling has easily occurred when 2 persons have turned on online meeting tools in the same room.

- Suddenly, a child of an online participant terminated mute and began talking when the participant went to the restroom (-> co-host was forcibly muted).

- Chair misses the order of presentation, however, many people wrongly recognize it as "presentation order has changed due to presentation cancellation:".

- Due to presentation mode problems, the presenter has not recognized that the slide has not turned over online even if the slide has turned over in local presentation mode, even if we prepared a guidebook.

- Zoom application is comparatively heavy application so that an old device (1st iPad mini) cannot decode movie on time and delay several tens of seconds.

- One participant prepared an avatar tool, however, if it operates with Zoom application, motion estimation function has sometimes outputs irregular estimation because of high CPU load.

After the meeting, many chairs talked that workload is much heavier than that of onsite because browsability of participants on online meeting was comparatively bad. Thus, it is important to prepare enough numbers of co-chairs who can help a chair.

\subsection{DEIM2020}

DEIM2020 ${ }^{17}$ was a workshop organized by the Data Engineering Research Committee of the Institute of Electronics, Information and Communication Engineers (IEICE) ${ }^{18}$, the Japan Database Society $(J D B S)^{19}$, and the SIG of DataBase Systems in the Information Processing Society of Japan (IPSJ) ${ }^{20}$ for the purpose of discussing and exchanging opinions on various research topics related to data engineering and information management. It is a camp-style workshop from 2-4 March and is an academic event of a size that attracted 574 participants in 2018 results; it will be held over several days with 10 or so parallel sessions; and it will be held at the same time, with a maximum attendance of 5,000 participants.

Meanwhile, at the National Institute of Informatics (NII), the director, who is also one of the people involved in the DEIM, asked several constituents on the morning of the 17 th if there was anyone who could help make the conference online, and the NII team was formed to discuss not only the DEIM, but also online workshops and lectures. The following outlines and policies were presented by the Director

- The significance of holding the meeting online is to avoid crowding.

- The main purpose of the conference is to provide the speakers with a valuable opportunity to present their research, and therefore the ability to ask and answer questions is essential.

- To ensure that the conference will be broadly usable, not just for information-related conferences, so that it can be used by people who are not familiar with information technology.

It was decided to outsource the development to software companies, and on the 18th the specifications were drawn up and inquiries were made to several companies, and on the 19th and 20th meetings were held with the DEIM2020 Executive Committee ("Executive Committee") via video conference. For the online event, the NII team felt that training of the chairperson, operators, and presenters was essential before the event, as well as documentation to facilitate the training. The Executive Committee committed to preparing these advance preparations within the Executive Committee. It was also decided at this meeting that the NII side would provide a room in the NII building for the operation. It was decided at the meeting that Webex Meetings, LINE LIVE ${ }^{21}$ and YouTube Live would be used in the production.

On the 21st February, a Webex Meetings concurrent connection experiment was conducted to investigate the problems with 100 concurrent connections. The problems that emerged from this survey are as follows.

(1) Handling of participants who entered the conference room with the microphone turned on.

(2) Notification sound when you enter a room.

(3) Degradation of sound and image quality dependent on the speaker's environment.

\footnotetext{
${ }^{17}$ DEIM2020 (Japanese) https://db-event.jpn.org/deim2020

${ }^{18}$ The Institute of Electronics, Information and Communication Engineers (IEICE) Technical Committee of Data Engineering (Japanese)

https://www.ieice.org/iss/de/jpn

${ }^{19}$ The Database Society of Japan https://dbsj.org/en

${ }^{20}$ Information Processing Society of Japan (IPSJ) SIG-DBS (DataBase System) (Japanese)

http://www.ipsj-dbs.org

${ }^{21}$ LINE LIVE https://linelive.me
} 
It was found that 1 and 2 could be handled by the Webex Meetings function; for 3 it was difficult to cope technically, so it was decided to notify the chairperson, operators and presenters to prepare a good quality headset microphone. In addition, the organizing committee requested that the number of participants in each conference room be obtained and visualized in real time. We searched for Webex Meetings XML API that has such a feature to meet this request, but we could not find an XML API Request that allows us to retrieve the number of participants in real time. We created a script that scraped the HTML of the conference room, counted the number of participants, and output it to Google Spreadsheet.

On-site work began four days before the day of the event (March 2), and LAN setup and continuity checks were carried out from Thursday, March 27. On the 28th, the consolidation of the equipment in the NII started. In the evening of the same day, a final check of the 10 parallels was conducted by the organizing committee, where the chairperson and speakers were connected to each conference room to share materials and, in particular, to check for possible obstacles that might occur when using the presenter's tools. These pre- and production findings were compiled by the Executive Committee and made available on GitHub.

On the 29th, the rental equipment was delivered and set up; on the 1st of March, the Secretariat was set up and instructions were given to the Chairperson and operators. The URLs of all Webex Meetings meeting rooms for the three days of DEIM2020 were issued on this day. These URLs were made available on a website that could only be viewed by those who had registered to attend and were anchored to the sessions listed in the program. On the physical front, alcohol disinfectant solutions and masks were prepared at the entrance to the relevant compartment and infrared thermometers were used to take regular non-contact body temperature readings of those entering and staying in the room.

The director has been quietly spreading the news to the media, and on the day of the event on March 2, several newspapers and TV stations visited to cover the event, with the Asahi Shimbun publishing an article on asahi.com that same day ${ }^{22}$ and the NHK News Web publishing an article early the next morning. NHK published an article on NHK NEWS WEB in the early morning of the next day ${ }^{23}$. The internet media outlet BUSINESS INSIDER also published an article in the evening of March 3rd. In addition, in the DBSJ Hour session on the first day of March 2, the NII team was thanked, and after DEIM 2020, the NII team was thanked on the DEIM website. While it may seem simple, the potential for such lyrical considerations to build a positive and evolving relationship between the Executive Committee and supporting organizations should not be ignored.

DEIM 2020 concluded on 4 March with 563 participants.

\subsection{The 82nd National Convention of the IPSJ}

The 82nd National Convention of Information Processing Society of Japan (IPSJ) was scheduled to be held at Kanazawa Institute of Technology from March 5 to 7, but was cancelled. The general

\footnotetext{
${ }^{22}$ Online conference, no concentrated contact now held for new pneumonia (Japanese) https:/www.asahi.com/articles/ASN326WBNN32ULBJ01G.html

${ }^{23}$ Development of a conference system for conferences. Hundreds of people can participate remotely on the Internet.

https://www3.nhk.or.jp/news/html/20200303/k10012310661000.html
}

and student sessions were held online on the same dates using the video conference service Zoom. The number of participants in this national conference usually exceeds 3,000 in previous years. The 82nd conference had 151 general and student sessions and 1,135 presentations, which were scheduled to be held in parallel over three days at 31 session sites.

How many systems are needed to hold an online conference with a nominal number of 3,000 participants? In the case of the IPSJ National Conference, the number of sessions and lectures is large, but the number of presentations per session is high 9. The time schedule for the online sessions is the same as the original program, and 31 parallel videoconference rooms are set up for each of the 31 session rooms. This means that about 30 sessions with about 30 participants per venue are running in about 30 parallel rooms at each time. This is not a scale that we need to worry about as a video conferencing service. Also, since the sessions are held entirely online, participants are spread out across the country, so there are no network bottlenecks ${ }^{24}$.

Another issue was the lack of support staff. Normally, a parttime student from the host university would be assigned to support the chairperson at each session venue. This time, however, in consideration of preventing the spread of a new type of coronavirus infection, it was decided that the operation of having dozens of part-time students gather in one place to work on the day of the event, as was the case with DEIM2020, should be strictly avoided. On the other hand, there was no time to make arrangements for equipment or provide advance training for part-time students to work online from their homes or other locations. The management of the sessions on the day was basically left to the chairperson, while the conference office worked behind the scenes with Zoom and telephone support (Figure 2).

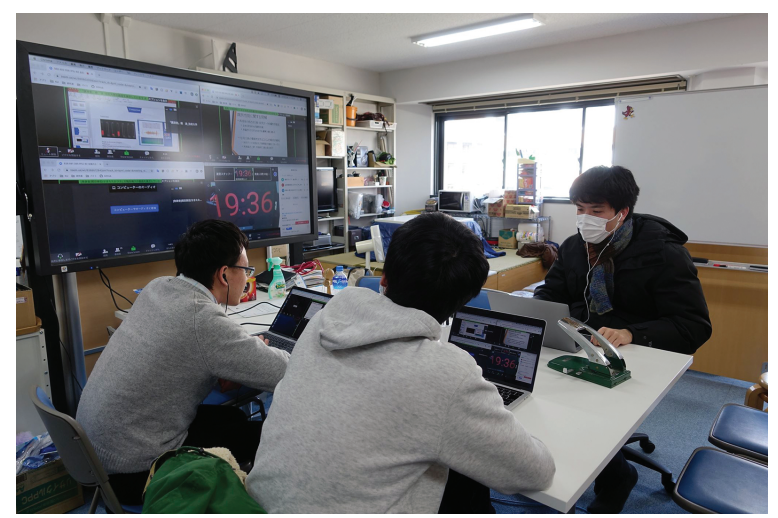

Figure 2: Support teams at Kyoto University

On reflection, we have written too much detail on the portal site documents. In particular, the "Guide to Connecting to the Conference on Information Processing Society of Japan Zoom" was very well received by those who are planning to hold an online conference in Zoom, but it was later found out that most of the

\footnotetext{
${ }^{24}$ Network bottlenecks in homes, apartment complexes, and mobile base stations will become apparent later, but only after universities start offering online lectures in the end of April.
} 
Hiroki Kashiwazaki, Takuro Ozaki, Hajime Shimada, Yusuke Komiya, Eisaku Sakane, Kazuhiro Mishima, Shiu Sakashita, Nariyoshi Yamai, Yoshiaki Kitaguchi, and Kensuke SIGUCCS '20, March 14-April 30, 2021, Virtual Event, USA

participants did not read it because it was too much information. On the other hand, the "Simple Manual for Audience and Presentations using Zoom" was easier to understand and better, since it contained only the minimum amount of information necessary to participate in the conference.

On March 4, the day before the conference started, the support conference room started. The participants were asked to confirm that they could connect to the conference room, check if the audio was muted, and that they could unmute and leave the room. Typical problems are as follows.

- Connection and image but no audio is available (i.e. "Connect Computer Audio" is not enabled)

- Unmuting causes howling (another device in the same room is producing sound and picking up the sound)

- Your voice is muffled when you speak (microphone is too far or poorly performing)

- Sound is cut off (Wi-Fi signal is weak)

- I can't share slides (I didn't check the "share screen" method in the simple manual)

- The PowerPoint presenter tool is displayed (you need to switch it when connecting to an external display).

- Audio played back on the computer is not transmitted

What I found out after participating in the test was that both the chairperson and the presenter seemed to have always tested Zoom for beginners. Thanks to this, there were almost no cases of not being able to connect to Zoom and not being able to present at the event. It was fortunate that the conference had its own test site, because people started to report on social networking sites that the test site was not working due to overload around this time.

By switching to online, only 753 of the 1135 presentations listed in the preliminary report were given online. The reasons for the lack of online presentations included: companies could not get permission to present online in time, and students could not prepare for their presentations with their instructors due to restrictions on their attendance at the university due to infectious diseases.

\section{DISCUSSION}

The following is a summary of the definition of the requirements for online meeting tools for academic research councils and their solutions. The study of the solution was realized by using other tools in combination.

(1) Difficulties for participants to be familiar with online meeting tools

It is easy to become proficient in online conferencing tools when meeting within an organization, or within a small number of organizations. This can be done by familiarizing yourself with the online meeting tools in advance of an offline meeting, or by preparing people from your organization to participate in an online meeting, who are proficient in the online meeting tools. However, in most cases, a small number of participants (often as few as one) from many organizations participate in a scientific research conference, and it is often impossible to receive instruction from a skilled person offline. Therefore, we provide sufficient opportunity to practice connecting to the online conference system and using the presentation mode prior to the conference. We also recommend practicing the presentation mode during the break before the session.

(2) Copyright Considerations

It is obvious that the observance of copyright is also important in academic presentations. When distributing online via the Internet, new considerations for the right of public transmission and the right to make transmittable are required, and these must be sufficiently informed to the authors. In addition, some organizations require consideration of the so-called "pseudo-copyright", and it is a burden for event organizers, who are not experts in copyright, to decide how far to go. It is hoped that a unified guideline for society will be established as soon as possible. In Japan, the Agency for Cultural Affairs is soliciting opinions on the "Draft Ministerial Ordinance for Amending Part of the Enforcement Regulations of the Copyright Act". In Japan, the "Public Transmission for Classroom Purposes Compensation System" was introduced on April 28, 2020. This system allows non-profit educational institutions to transmit works to the public for classroom purposes if they pay a certain amount of compensation ${ }^{25}$.

(3) Allowing offline to discuss after the conference. It is significant to present at a research conference not only in the formal question and answer period but also in the break time after the presentation. These discussions cover a wide variety of topics, including discussions based on questions that come up after the question and answer period, discussions that do not fit in the formal question and answer period, frank discussions that are a bit more reserved in the formal question and answer period, and discussions in narrower areas such as personal interests. We felt that this opportunity would be lost if the conference were to go online. Although many online conferencing tools have a chat function as shown in Table 1 , it is difficult to continue the discussion as soon as the meeting ends. For this reason, we considered using slack, a chat-based online communication tool, in conjunction with the conference, to enable discussions for about a week after the conference.

\section{CONCLUSION}

Four operational examples of online academic conferences held in Japan at the end of February and early March 2020 are described in the paper. And as of November 2020, more and more different online conferencing tools or combinations of them are in use. The purpose of this paper is to document the trials and tribulations of operational staff during the first phase of disaster response. We hope that this paper will help operations staff to prepare for future disasters.

\footnotetext{
${ }^{25}$ Compensation System for Public Transmission for Educational Purposes https://sartras.or.jp/en
} 